\title{
THERMOELASTIC STRAIN AND STRESS FIELDS DUE TO A SPHERICAL INCLUSION IN AN ELASTIC HALF-SPACE
}

\author{
Kuldip Singh, Renu Muwal \\ Guru Jambheshwar University of Science and Technology, Department of Mathematics, Hisar, Haryana, India \\ e-mail: renumuwal66@gmail.com
}

\begin{abstract}
In this paper, closed form analytical expressions for thermoelastic strain and stress components due to a spherical inclusion in an elastic half-space are obtained. These expressions are derived in the context of steady-state uncoupled thermoelasticity using thermoelastic displacement potential functions. The thermal strain and stress fields are generated due to differences in the coefficients of linear thermal expansion between a subregion and the surrounding material. The strain and stress components for exterior points of the spherical inclusion are same as those of the center of dilatation. Variations of strain and stress components for exterior and interior points of the spherical inclusion are shown graphically.
\end{abstract}

Keywords: uncoupled thermoelasticity, strain and stress fields, potential functions, spherical inclusion

\section{Introduction}

Thermoelasticity is an extension of theory of elasticity to include thermal effects. Theory of thermoelasticity is concerned with the interaction between the thermal field and elastic bodies. Comprehensive treatises on the topics of thermoelasticity have been covered in the classical texts (Nowinski, 1978; Truesdell, 1984; Nowacki, 1986; Boley and Weiner, 1997; Boresi et al., 2011; etc.). The study of thermoelasticity has begun with Duhamel (1837) and Neumann (1885), who postulated equations of linear thermoelasticity for isotropic bodies. Goodier (1937) studied the static problem of uncoupled thermoelasticity by employing the method of superposition using displacement potential functions. With the help of Goodier's method, Mindlin and Cheng (1950) obtained the thermal stress in a semi-infinite elastic solid with the traction free surface for the centre of dilatation using Galerkin's vector stress function. The result was then applied to the case of a spherical inclusion in a semi-infinite medium. Nowacki (1957) considered the problem of an instantaneous source of heat in an infinite isotropic elastic space and determined the state of stress using the potential of thermoelastic strain. Also, stress components in a semi-infinite elastic space due to an instantaneous source of heat was obtained using the method of Galerkin's displacement function and the Fourier integral under stress free surface boundary conditions. Sen (1957) presented a direct method for solving problems of circular holes in an isotropic elastic plate having prescribed displacements on the edge. The problem of deformation in an infinite isotropic plate due to the centre of dilatation in the form of a nucleus of thermoelastic strain at a finite distance from the hole was also discussed. Sharma (1957) obtained deformation and stress fields due to a nucleus of thermoelastic strain in an infinite solid having a spherical cavity using interior and exterior spherical harmonics. Another problem of a solid sphere at zero temperature having a heated nucleus inside was also discussed. Biharmonic solutions to steady state thermoelastic problems in three dimensions were derived by Nowinski (1961). Nowinski (1963) proved a mean value theorem for an arbitrary steady-state thermoelastic problem for an 
isotropic elastic solid sphere. The stress components at the center of the sphere were expressed in terms of temperature on the surface of the sphere.

Guell and Dundurs (1967) obtained elastic fields due to a subregion between two joined half-spaces by differentiation from the corresponding fields for the whole space using the solutions for the center of dilatation. The Papkovich-Neuber displacement potentials were used when the interface between two joined half-spaces was a smooth interface and perfectly bonded. Those results were then applied to a spherical inclusion. The case of the force on the center of dilatation was also discussed. $\mathrm{Hu}$ (1989) considered the problem of a parallelepipedic thermal inclusion in a three-dimensional half-space and derived analytical solution using Goodier's method of the nucleus of thermal strain or the center of dilatation. Wang and Huang (1991) studied some thermoelastic problems in the half-space by using Goodier's thermoelastic potential functions and general solutions of elasticity based on the Boussinesq solutions (consisting of different harmonic functions). Yu et al. (1992) and Yu and Sanday (1992), respectively, derived analytical solutions for thermoelastic displacements and stresses due to an inclusion in a dissimilar medium consisting of two joined semi-infinite elastic solids and for the centre of dilatation in a plate. The stress-deformation state of an elastic half-space due to an inhomogeneous spheroidal thermal inclusion in the form of a prolate or oblate ellipsoid of revolution under stress-free surface boundary conditions was discussed by Kolesov et al. (1992) using thermoelastic displacement potential functions and the Teredzave method. Yu et al. (2002) calculated thermal stresses induced by an ellipsoidal inclusion with uniform dilatational eigenstrains in one of the two perfectly bonded semi-infinite solids using the method of dilatation centres and potential functions. Davies (2003) derived the elastic field due to a non-uniform temperature or a coherently misfitting inclusion in a semi-infinite region from the corresponding field in an infinite region.

Using Green's function method and series expansion techniques, closed form solutions for displacement and stress fields due to a hemispherical inclusion with a uniform eigenstrain in a semi-infinite isotropic elastic medium were obtained by Linzhi (2003). Liu and Wang (2005) obtained the elastic field caused by eigenstrains in a half-space using two types of numerical techniques - discrete correlation and fast Fourier transform as well as discrete convolution and fast Fourier transform. To obtain that field, analytical solutions for influence coefficients were expressed in terms of derivatives of four key integrals. Zhou et al. (2009) introduced a fast method for solving the problem of three-dimensional arbitrarily shaped inclusions in an isotropic half-space using a combination of 2D and 3D fast Fourier transform. A fast method for solving contact problems for a half-space containing multiple inhomogeneities such as voids, cavities, inclusions and fibers was presented by Leroux et al. (2010). The displacement and stress fields due to eigenstrains of all spherical inclusion were obtained using Eshelby's equivalent inclusion method along with 2D and 3D fast Fourier transforms. Itou (2014) derived basic equations for thermoelastic plane stress conditions and thermoelastic plane strain conditions. Two problems were solved using thermoelastic displacement potential functions: (i) axisymmetric thermal stresses for a hollow thin disk, (ii) thermal stresses for an infinite thin plate with a circular hole under uniform heat flow. Kumagai et al. (2014) presented different representations of a seismic spherical source. Those different representations consisted of a spherical source (S1), spherical crack source (S2), isotropic source of three mutually perpendicular vector dipoles (S3) or three mutually perpendicular tensile cracks (S4), Eshelby's spherical source with stress-free strain (S5) and Eshelby's spherical source with strain-free stress (S6). Static displacement fields due to those sources in an infinite medium and in a half-space were also derived.

In the present paper, we obtain thermoelastic strain and stress fields due to a spherical inclusion in an elastic half-space in the context of steady-state uncoupled thermoelasticity. Following the method opted by Davies (2003), we derive thermoelastic strain and stress components for an infinite region from which corresponding fields can be derived in the semi-infinite region. The expressions for these fields are obtained for the axisymmetric problem in cylindrical coordinates 
using thermoelastic displacement potential functions. These results are in good agreement with the results found by Mindlin and Cheng (1950).

\section{Theory}

In the linear theory of thermoelasticity, the total strain can be written as the sum of mechanical and thermal strains (Goodier, 1937; and Nowinski, 1978)

$$
e_{i j}=e_{i j}^{(M)}+e_{i j}^{(T)}
$$

in which for an isotropic material, the thermal strain takes the form $e_{i j}^{(T)}=\alpha T \delta_{i j}$, where $\alpha$ is the coefficient of linear thermal expansion, $T$ is temperature difference, $\delta_{i j}$ is Kronecker delta. Then in the context of uncoupled linear thermoelasticity, the displacement-temperature equation of equilibrium in the absence of body forces can be written as

$$
\nabla^{2} \mathbf{u}+\frac{1}{1-2 \nu} \nabla(\nabla \cdot \mathbf{u})=\frac{2(1+\nu)}{1-2 \nu} \alpha \nabla T
$$

where $e_{i j}$ are components of the strain tensor; $\mathbf{u}$ is the displacement vector and $\nu$ is Poisson's ratio.

The uncoupled heat conduction equation for a steady state temperature field $T$ with $Q$ as the heat supply and $\lambda_{0}$ as the thermal conductivity can be written as

$$
\nabla^{2} T=\frac{-Q}{\lambda_{0}}
$$

The solution to inhomogeneous equation (2.2) can be expressed as

$$
\mathbf{u}=\mathbf{u}^{c}+\mathbf{u}^{p}
$$

where $\mathbf{u}^{c}$ is the complementary function satisfying the homogeneous equation of (2.2) and $\mathbf{u}^{p}$ represents the particular solution to the displacement field generated by the temperature field $T$.

According to Goodier's method (Timoshenko and Goodier, 1951), the displacement $\mathbf{u}^{(\infty)}(\mathbf{r})$ for an infinite solid is given by $\mathbf{u}^{(\infty)}=\nabla \varphi$, where the potential function $\varphi$ satisfies Poisson's equation

$$
\nabla^{2} \varphi=\frac{1+\nu}{1-\nu} \alpha T(\mathbf{r})
$$

Then the function $\varphi$ is obtained as

$$
\varphi(\mathbf{r})=\frac{-1}{4 \pi} \frac{1+\nu}{1-\nu} \alpha \int \frac{T\left(\mathbf{r}^{\prime}\right)}{\left|\mathbf{r}-\mathbf{r}^{\prime}\right|} d^{3}\left(\mathbf{r}^{\prime}\right)
$$

where $\left|\mathbf{r}-\mathbf{r}^{\prime}\right|=|(x, y, z)-(\xi, \eta, \varsigma)|=\sqrt{(x-\xi)^{2}+(y-\eta)^{2}+(z-\varsigma)^{2}}$ is the distance between the points $(x, y, z)$ and $(\xi, \eta, \varsigma)$.

Then the displacement, strain and stress components in the cylindrical polar coordinates $(r, \theta, z)$ are expressed in terms of the potential function $\varphi$ as (Barber, 2002; Sadd, 2005)

$$
u_{r}=\frac{\partial \varphi}{\partial r} \quad u_{\theta}=\frac{1}{r} \frac{\partial \varphi}{\partial \theta} \quad u_{z}=\frac{\partial \varphi}{\partial z}
$$

and

$$
\begin{aligned}
e_{r r}=\frac{\partial u_{r}}{\partial r} & e_{\theta \theta}=\frac{1}{r}\left(u_{r}+\frac{\partial u_{\theta}}{\partial \theta}\right) & e_{r \theta}=\frac{1}{2}\left(\frac{1}{r} \frac{\partial u_{r}}{\partial \theta}+\frac{\partial u_{\theta}}{\partial r}-\frac{u_{\theta}}{r}\right) \\
e_{z z}=\frac{\partial u_{z}}{\partial z} & e_{\theta z}=\frac{1}{2}\left(\frac{\partial u_{\theta}}{\partial z}+\frac{1}{r} \frac{\partial u_{z}}{\partial \theta}\right) & e_{z r}=\frac{1}{2}\left(\frac{\partial u_{r}}{\partial z}+\frac{\partial u_{z}}{\partial r}\right)
\end{aligned}
$$


and

$$
\begin{aligned}
& \frac{1}{2 \mu} \sigma_{r r}=\frac{\partial^{2} \varphi}{\partial r^{2}}-\nabla^{2} \varphi \quad \frac{1}{2 \mu} \sigma_{\theta \theta}=\frac{1}{r} \frac{\partial \varphi}{\partial r}+\frac{1}{r^{2}} \frac{\partial^{2} \varphi}{\partial \theta^{2}}-\nabla^{2} \varphi \quad \frac{1}{2 \mu} \sigma_{z z}=\frac{\partial^{2} \varphi}{\partial z^{2}}-\nabla^{2} \varphi \\
& \frac{1}{2 \mu} \sigma_{r \theta}=\frac{\partial}{\partial r}\left(\frac{1}{r} \frac{\partial \varphi}{\partial \theta}\right) \quad \frac{1}{2 \mu} \sigma_{\theta z}=\frac{1}{r} \frac{\partial^{2} \varphi}{\partial \theta \partial z} \quad \frac{1}{2 \mu} \sigma_{z r}=\frac{\partial^{2} \varphi}{\partial z \partial r}
\end{aligned}
$$

where the Laplacian operator in the cylindrical polar coordinates $(r, \theta, z)$ is given by

$$
\nabla^{2} \varphi=\left(\frac{\partial^{2}}{\partial r^{2}}+\frac{1}{r} \frac{\partial}{\partial r}+\frac{1}{r^{2}} \frac{\partial^{2}}{\partial \theta^{2}}+\frac{\partial^{2}}{\partial z^{2}}\right) \varphi
$$

\section{Formulation and solution of the problem}

We consider an axisymmetric problem of a spherical inclusion in the upper half-space $(z \geqslant 0)$ having a different coefficient of thermal expansion to that of the half-space but the same elastic constants as in Wang and Huang (1991). Due to this difference in the coefficients of thermal expansion between the sub region and its surrounding material, say $\eta_{0}$, a thermoelastic stress field is generated. Let radius of the spherical inclusion be $a$ and center of it be located at the point $r=0$ and $z=h$, where $h>a$, as shown in Fig. 1 . The surface $z=0$ is taken as traction free surface. We take the axis of symmetry as the $z$ axis, then for the axisymmetric problem in the cylindrical coordinates $(r, \theta, z)$, all quantities are independent of $\theta$. Then using Eq. (2.7), the displacement vector $\mathbf{u}$ has the form $\mathbf{u}=\left(u_{r}, 0, u_{z}\right)$. Therefore, the components of the strain and stress tensor can be written in the following form, see equations (2.8) and (2.9)

$$
\begin{array}{ll}
e_{r r}=\frac{\partial u_{r}}{\partial r} \quad e_{\theta \theta}=\frac{u}{r} & e_{z z}=\frac{\partial u_{z}}{\partial z} \\
e_{z r}=\frac{1}{2}\left(\frac{\partial u_{r}}{\partial z}+\frac{\partial u_{z}}{\partial r}\right) & e_{r \theta}=0 \quad e_{\theta z}=0
\end{array}
$$

and

$$
\begin{array}{llrl}
\frac{1}{2 \mu} \sigma_{r r}=\frac{\partial^{2} \varphi}{\partial r^{2}}-\nabla^{2} \varphi & \frac{1}{2 \mu} \sigma_{\theta \theta}=\frac{1}{r} \frac{\partial \varphi}{\partial r}-\nabla^{2} \varphi & \frac{1}{2 \mu} \sigma_{z z}=\frac{\partial^{2} \varphi}{\partial z^{2}}-\nabla^{2} \varphi \\
\frac{1}{2 \mu} \sigma_{z r}=\frac{\partial^{2} \varphi}{\partial z \partial r} \quad \sigma_{r \theta}=0 \quad \sigma_{\theta z}=0 &
\end{array}
$$

where

$$
\nabla^{2} \varphi=\left(\frac{\partial^{2}}{\partial r^{2}}+\frac{1}{r} \frac{\partial}{\partial r}+\frac{\partial^{2}}{\partial z^{2}}\right) \varphi
$$

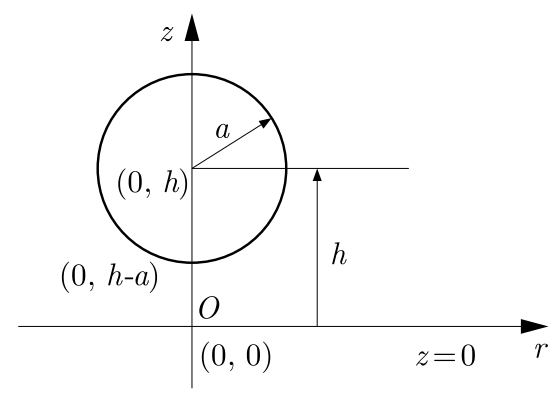

Fig. 1. A spherical inclusion in a thermoelastic half-space 
Then according to Wang and Huang (1991), the thermoelastic potential function $\varphi$ satisfies the following Poisson's equations, when temperature of the semi-infinite region increases up to $T_{0}$

$$
\nabla^{2} \varphi=\left\{\begin{array}{lll}
\frac{1+\nu}{1-\nu} \alpha T=\frac{1+\nu}{1-\nu} \eta_{0} T_{0} & \text { for } & R_{1} \leqslant a \\
0 & \text { for } & R_{1}>a
\end{array}\right.
$$

where $R_{1}^{2}=r^{2}+(z-h)^{2}$ is the distance of the point $(r, z)$ from $(0, h)$. Then, the function $\varphi$ for this problem is taken as (Wang and Huang 1991)

$$
\varphi=\left\{\begin{array}{lll}
\frac{1}{6} K R_{1}^{2} & \text { for } & R_{1} \leqslant a \\
\frac{1}{6} K\left(3 a^{2}-2 \frac{a^{3}}{R_{1}}\right) & \text { for } & R_{1}>a
\end{array}\right.
$$

where

$$
K=\frac{1+\nu}{1-\nu} \eta_{0} T_{0}
$$

Considering the boundary conditions, we assume that the boundary $z=0$ of the half-space is traction free surface i.e.

$$
\sigma_{z z}=\sigma_{r z}=0 \quad \text { at } \quad z=0
$$

Now according to Davies (2003), the strain and stress components within the semi-infinite region $z \geqslant 0$ with the traction free surface in terms of strain and stress components for an infinite region for the axisymmetric problem in the $r z$-plane can be reduced to the form

$$
\begin{array}{ll}
e_{r r}=e_{r r}^{(\infty)}+(3-4 \nu) \bar{e}_{r r}^{(\infty)}+2 z \frac{\partial}{\partial z} \bar{e}_{r r}^{(\infty)} & e_{\theta \theta}=e_{\theta \theta}^{(\infty)}+(3-4 \nu) \bar{e}_{\theta \theta}^{(\infty)}+2 z \frac{\partial}{\partial z} \bar{e}_{\theta \theta}^{(\infty)} \\
e_{z z}=e_{z z}^{(\infty)}-(1-4 \nu) \bar{e}_{z z}^{(\infty)}+2 z \frac{\partial}{\partial z} \bar{e}_{z z}^{(\infty)} & e_{r z}=e_{r z}^{(\infty)}-\bar{e}_{r z}^{(\infty)}-2 z \frac{\partial}{\partial z} \bar{e}_{r z}^{(\infty)} \\
e_{r \theta}=0=e_{\theta z} &
\end{array}
$$

and

$$
\begin{aligned}
& \sigma_{r r}=\sigma_{r r}^{(\infty)}+(3-4 \nu) \bar{\sigma}_{r r}^{(\infty)}-4 \nu \bar{\sigma}_{z z}^{(\infty)}+2 z \frac{\partial}{\partial z} \bar{\sigma}_{r r}^{(\infty)} \\
& \sigma_{\theta \theta}=\sigma_{\theta \theta}^{(\infty)}+(3-4 \nu) \bar{\sigma}_{\theta \theta}^{(\infty)}-4 \nu \bar{\sigma}_{z z}^{(\infty)}+2 z \frac{\partial}{\partial z} \bar{\sigma}_{\theta \theta}^{(\infty)} \quad \sigma_{r \theta}=0=\sigma_{\theta z} \\
& \sigma_{z z}=\sigma_{z z}^{(\infty)}-\bar{\sigma}_{z z}^{(\infty)}+2 z \frac{\partial}{\partial z} \bar{\sigma}_{z z}^{(\infty)} \quad \sigma_{r z}=\sigma_{r z}^{(\infty)}-\bar{\sigma}_{r z}^{(\infty)}-2 z \frac{\partial}{\partial z} \bar{\sigma}_{r z}^{(\infty)}
\end{aligned}
$$

Then the displacement, strain and stress fields in the infinite region and those at the image point for exterior points $\left(R_{1}>a\right)$ of the spherical inclusion (where $\nabla^{2} \varphi=0$ ) are obtained using $\mathbf{u}^{(\infty)}=\nabla \varphi$ and equations (3.1)-(3.3)

$$
\begin{aligned}
& u_{r}^{(\infty)}=\frac{\partial \varphi}{\partial r}=\frac{1}{3} K a^{3}\left(\frac{r}{R_{1}^{3}}\right) \quad u_{\theta}^{(\infty)}=\frac{1}{r} \frac{\partial \varphi}{\partial \theta}=0 \quad u_{z}^{(\infty)}=\frac{\partial \varphi}{\partial z}=\frac{1}{3} K a^{3}\left(\frac{z-h}{R_{1}^{3}}\right) \\
& e_{r r}^{(\infty)}=\frac{\partial^{2} \varphi}{\partial r^{2}}=\frac{1}{3} K a^{3}\left(\frac{1}{R_{1}^{3}}-\frac{3 r^{2}}{R_{1}^{5}}\right) \quad e_{\theta \theta}^{(\infty)}=\frac{u_{r}}{r}=\frac{1}{3} K a^{3}\left(\frac{1}{R_{1}^{3}}\right) \quad e_{r \theta}^{(\infty)}=e_{\theta z}^{(\infty)}=0 \\
& e_{z z}^{(\infty)}=\frac{\partial^{2} \varphi}{\partial z^{2}}=\frac{1}{3} K a^{3}\left(\frac{1}{R_{1}^{3}}-\frac{3(z-h)^{2}}{R_{1}^{5}}\right) \quad e_{r z}^{(\infty)}=e_{z r}^{(\infty)}=\frac{\partial^{2} \varphi}{\partial r \partial z}=-K a^{3} \frac{r(z-h)}{R_{1}^{5}}
\end{aligned}
$$




$$
\begin{aligned}
& \frac{1}{2 \mu} \sigma_{r r}^{(\infty)}=\frac{1}{3} K a^{3}\left(\frac{1}{R_{1}^{3}}-\frac{3 r^{2}}{R_{1}^{5}}\right) \quad \frac{1}{2 \mu} \sigma_{\theta \theta}^{(\infty)}=\frac{1}{3} K a^{3}\left(\frac{1}{R_{1}^{3}}\right) \quad \sigma_{r \theta}^{(\infty)}=0=\sigma_{\theta z}^{(\infty)} \\
& \frac{1}{2 \mu} \sigma_{z z}^{(\infty)}=\frac{1}{3} K a^{3}\left(\frac{1}{R_{1}^{3}}-\frac{3(z-h)^{2}}{R_{1}^{5}}\right) \\
& \bar{u}_{r}^{(\infty)}=\frac{1}{3} K a^{3}\left(\frac{r}{R_{2}^{3}}\right) \quad \frac{1}{2 \mu} \sigma_{r z}^{(\infty)}=-K a^{3} \frac{r(z-h)}{R_{1}^{5}} \\
& \bar{e}_{r r}^{(\infty)}=\frac{1}{3} K a^{3}\left(\frac{1}{R_{2}^{3}}-\frac{3 r^{2}}{R_{2}^{5}}\right) \quad \bar{u}_{z}^{(\infty)}=\frac{-1}{3} K a^{3}\left(\frac{z+h}{R_{2}^{3}}\right) \\
& \bar{e}_{z z}^{(\infty)}=\frac{1}{3} K a^{3}\left(\frac{1}{R_{2}^{3}}-\frac{3(z+h)^{2}}{R_{2}^{5}}\right) \quad \bar{e}_{\theta \theta}^{(\infty)}=\frac{1}{3} K a^{3}\left(\frac{1}{R_{2}^{3}}\right) \quad \bar{e}_{r \theta}^{(\infty)}=\bar{e}_{\theta z}^{(\infty)}=0 \\
& \frac{1}{2 \mu} \bar{\sigma}_{r r}^{(\infty)}=\frac{1}{3} K a^{3}\left(\frac{1}{R_{2}^{3}}-\frac{3 a^{3}}{R_{2}^{5}}\right) \\
& \frac{1}{2 \mu} \bar{\sigma}_{z z}^{(\infty)}=\frac{1}{3} K a^{3}\left(\frac{1}{R_{2}^{3}}-\frac{3(z+h)}{R_{2}^{5}}\right) \quad \frac{1}{2 \mu} \bar{\sigma}_{\theta \theta}^{(\infty)}=\frac{1}{3} K a^{3}\left(\frac{1}{R_{2}^{3}}\right) \\
& \frac{1}{2 \mu} \bar{\sigma}_{r z}^{(\infty)}=K a^{3} \frac{r(z+h)}{R_{2}^{5}}
\end{aligned}
$$

where $(0,-h)$ is the image of point $(0, h)$ and $R_{2}^{2}=r^{2}+(z+h)^{2}$ is the distance of the point $(r, z)$ from $(0,-h)$.

Substituting equations (3.11), (3.14) and (3.12), (3.15) into (3.8) and (3.9), respectively, the strain and stress components in the thermoelastic half-space for exterior points $\left(R_{1}>a\right)$ of the spherical inclusion can be expressed as

$$
\begin{aligned}
& e_{r r}=\frac{1}{3} K a^{3}\left[\frac{1}{R_{1}^{3}}+\frac{3-4 \nu}{R_{2}^{3}}-\frac{6 z(z+h)}{R_{2}^{5}}-3 r^{2}\left(\frac{1}{R_{1}^{5}}+\frac{3-4 \nu}{R_{2}^{5}}-\frac{10 z(z+h)}{R_{2}^{7}}\right)\right] \\
& e_{\theta \theta}=\frac{1}{3} K a^{3}\left(\frac{1}{R_{1}^{3}}+\frac{3-4 \nu}{R_{2}^{3}}-\frac{6 z(z+h)}{R_{2}^{5}}\right) \\
& e_{z z}=\frac{1}{3} K a^{3}\left(\frac{1}{R_{1}^{3}}-\frac{1-4 \nu}{R_{2}^{3}}-\frac{18 z(z+h)}{R_{2}^{5}}-\frac{3(z-h)^{2}}{R_{1}^{5}}+\frac{3(1-4 \nu)(z+h)^{2}}{R_{2}^{5}}+\frac{30 z(z+h)^{3}}{R_{2}^{7}}\right) \\
& e_{r z}=-K a^{3} r\left(\frac{z-h}{R_{1}^{5}}+\frac{3 z+h}{R_{2}^{5}}-\frac{10 z(z+h)^{2}}{R_{2}^{7}}\right)
\end{aligned}
$$

and

$$
\begin{aligned}
& \sigma_{r r}=2 \mu \frac{K a^{3}}{3}\left[\frac{1}{R_{1}^{3}}+\frac{3-8 \nu}{R_{2}^{3}}-\frac{6 z(z+h)}{R_{2}^{5}}+\frac{12 \nu(z+h)^{2}}{R_{2}^{5}}-3 r^{2}\left(\frac{1}{R_{1}^{5}}+\frac{3-4 \nu}{R_{2}^{5}}-\frac{10 z(z+h)}{R_{2}^{7}}\right)\right] \\
& \sigma_{\theta \theta}=2 \mu \frac{K a^{3}}{3}\left(\frac{1}{R_{1}^{3}}+\frac{3-8 \nu}{R_{2}^{3}}-\frac{6 z(z+h)}{R_{2}^{5}}+\frac{12 \nu(z+h)^{2}}{R_{2}^{5}}\right) \\
& \sigma_{z z}=2 \mu \frac{K a^{3}}{3}\left(\frac{1}{R_{1}^{3}}-\frac{1}{R_{2}^{3}}-\frac{18 z(z+h)}{R_{2}^{5}}-\frac{3(z-h)^{2}}{R_{1}^{5}}+\frac{3(z+h)^{2}}{R_{2}^{5}}+\frac{30 z(z+h)^{3}}{R_{2}^{7}}\right) \\
& \sigma_{r z}=-2 \mu K a^{3} r\left(\frac{z-h}{R_{1}^{5}}+\frac{3 z+h}{R_{2}^{5}}-\frac{10 z(z+h)^{2}}{R_{2}^{7}}\right)
\end{aligned}
$$

Further, from equation (3.17), we can see that $\sigma_{z z}=\sigma_{r z}=0$ at the boundary $z=0$ of the half-space, which is in accordance with the boundary conditions as in equation (3.7). (1950)

Also, for the interior points $\left(R_{1} \leqslant a\right)$ of the spherical inclusion, as in Mindlin and Cheng

$$
\mathbf{u}_{i n t}=\mathbf{u}_{e x t}+\frac{a^{3} K \mathbf{R}_{1}}{3}\left(\frac{1}{a^{3}}-\frac{1}{R_{1}^{3}}\right)
$$


Using this, the following relations between the strain components of the exterior points $\left(R_{1}>a\right)$ and the interior points $\left(R_{1} \leqslant a\right)$ of the spherical inclusion are given by

$$
\begin{array}{rc}
e_{r r}^{i n t}=e_{r r}^{e x t}+\frac{K a^{3}}{3}\left(\frac{1}{a^{3}}-\frac{1}{R_{1}^{3}}\right)+K a^{3} \frac{r^{2}}{R_{1}^{5}} & e_{\theta \theta}^{i n t}=e_{\theta \theta}^{e x t}+\frac{K a^{3}}{3}\left(\frac{1}{a^{3}}-\frac{1}{R_{1}^{3}}\right) \\
e_{z z}^{i n t}=e_{z z}^{e x t}+\frac{K a^{3}}{3}\left(\frac{1}{a^{3}}-\frac{1}{R_{1}^{3}}\right)+K a^{3} \frac{(z-h)^{2}}{R_{1}^{5}} & e_{r z}^{i n t}=e_{r z}^{e x t}+K a^{3} \frac{r(z-h)}{R_{1}^{5}}
\end{array}
$$

Also, the relations between the stress components of the exterior points $\left(R_{1}>a\right)$ and the interior points $\left(R_{1} \leqslant a\right)$ of the spherical inclusion are given below, see Guell and Dundurs (1967)

$$
\begin{array}{lrl}
\sigma_{r r}^{i n t}=\sigma_{r r}^{e x t}-2 \mu K\left(\frac{2}{a^{3}}+\frac{1}{R_{1}^{3}}-\frac{3 r^{2}}{R_{1}^{5}}\right) & \sigma_{\theta \theta}^{i n t}=\sigma_{\theta \theta}^{e x t}-2 \mu K\left(\frac{2}{a^{3}}+\frac{1}{R_{1}^{3}}\right) \\
\sigma_{z z}^{i n t}=\sigma_{z z}^{e x t}-2 \mu K\left(\frac{2}{a^{3}}+\frac{1}{R_{1}^{3}}-\frac{3(z-h)^{2}}{R_{1}^{5}}\right) & \sigma_{r z}^{i n t}=\sigma_{r z}^{e x t}+6 \mu K \frac{r(z-h)}{R_{1}^{5}}
\end{array}
$$

Substituting equations (3.16) and (3.17) into (3.19) and (3.20), respectively, the strain and stress components in the thermoelastic half-space for the interior points $\left(R_{1} \leqslant a\right)$ of the spherical inclusion can be expressed as

$$
\begin{aligned}
& e_{r r}=\frac{1}{3} K a^{3}\left[\frac{1}{a^{3}}+\frac{3-4 \nu}{R_{2}^{3}}-\frac{6 z(z+h)}{R_{2}^{5}}-3 r^{2}\left(\frac{3-4 \nu}{R_{2}^{5}}-\frac{10 z(z+h)}{R_{2}^{7}}\right)\right] \\
& e_{\theta \theta}=\frac{1}{3} K a^{3}\left(\frac{1}{a^{3}}+\frac{3-4 \nu}{R_{2}^{3}}-\frac{6 z(z+h)}{R_{2}^{5}}\right) \\
& e_{z z}=\frac{1}{3} K a^{3}\left(\frac{1}{a^{3}}-\frac{1-4 \nu}{R_{2}^{3}}-\frac{18 z(z+h)}{R_{2}^{5}}+\frac{3(1-4 \nu)(z+h)^{2}}{R_{2}^{5}}+\frac{30 z(z+h)^{3}}{R_{2}^{7}}\right) \\
& e_{r z}=-K a^{3} r\left(\frac{3 z+h}{R_{2}^{5}}-\frac{10 z(z+h)^{2}}{R_{2}^{7}}\right)
\end{aligned}
$$

and

$$
\begin{aligned}
& \sigma_{r r}=2 \mu \frac{K a^{3}}{3}\left[\frac{-2}{a^{3}}+\frac{3-8 \nu}{R_{2}^{3}}-\frac{6 z(z+h)}{R_{2}^{5}}+\frac{12 \nu(z+h)^{2}}{R_{2}^{5}}-3 r^{2}\left(\frac{3-4 \nu}{R_{2}^{5}}-\frac{10 z(z+h)}{R_{2}^{7}}\right)\right] \\
& \sigma_{\theta \theta}=2 \mu \frac{K a^{3}}{3}\left(\frac{-2}{a^{3}}+\frac{3-8 \nu}{R_{2}^{3}}-\frac{6 z(z+h)}{R_{2}^{5}}+\frac{12 \nu(z+h)^{2}}{R_{2}^{5}}\right) \\
& \sigma_{z z}=2 \mu \frac{K a^{3}}{3}\left(\frac{-2}{a^{3}}-\frac{1}{R_{2}^{3}}-\frac{18 z(z+h)}{R_{2}^{5}}+\frac{3(z+h)^{2}}{R_{2}^{5}}+\frac{30 z(z+h)^{3}}{R_{2}^{7}}\right) \\
& \sigma_{r z}=-2 \mu K a^{3} r\left(\frac{3 z+h}{R_{2}^{5}}-\frac{10 z(z+h)^{2}}{R_{2}^{7}}\right)
\end{aligned}
$$

The results obtained above are in good agreement to those of Mindlin and Cheng (1950) for the spherical inclusion in the interior of a thermoelastic semi-infinite solid using the usual thermoelastic relation.

\section{Numerical results and discussion}

In this Section, graphical representations of the strain and stress components at the points $(0,0)$ and $(0, h-a)$ just outside the spherical inclusion in the thermoelastic half-space are obtained using MATLAB software programming. The numerical computations are carried out for Poisson's ratios $\nu=0.1$ to 0.5 . Figures $2 \mathrm{a}$ and $2 \mathrm{~b}$ respectively show the variation of strain 
components $e_{r r}\left(=e_{\theta \theta}\right)$ and $e_{z z}$ at the point $(0,0)$ for exterior points of the spherical inclusion along the distance and varying Poisson's ratio from 0.1 to 0.5 . From Fig. $2 \mathrm{a}$, we observe that the strain $e_{r r}\left(=e_{\theta \theta}\right)$ decreases smoothly with increasing values of the distance $h / a$, and it vanishes at infinitely large values of $h / a$. Also, as Poisson's ratio $\nu$ increases from 0.1 to 0.5 , this strain increases quantitatively. From Fig. 2b, we observe that the strain $e_{z z}$ assumes negative values for all the values of Poisson's ratio $\nu$ ranging from 0.1 to 0.5 . Further, it continuously increases with the increasing distance $h / a$ assuming the value of zero at infinitely large values of $h / a$. In Figs. 3a and 3b, respectively, the variation of strain components $e_{r r}\left(=e_{\theta \theta}\right)$ and $e_{z z}$ at the point $(0, h-a)$ just outside the spherical inclusion are presented for exterior points. From Fig. 3b, it can be seen that the strain $e_{z z}$ first increases and then decreases continuously as the distance $h / a$ increases. Further, this strain approaches a finite negative value (depending on Poisson's ratio) at infinitely large values of the distance $h / a$.
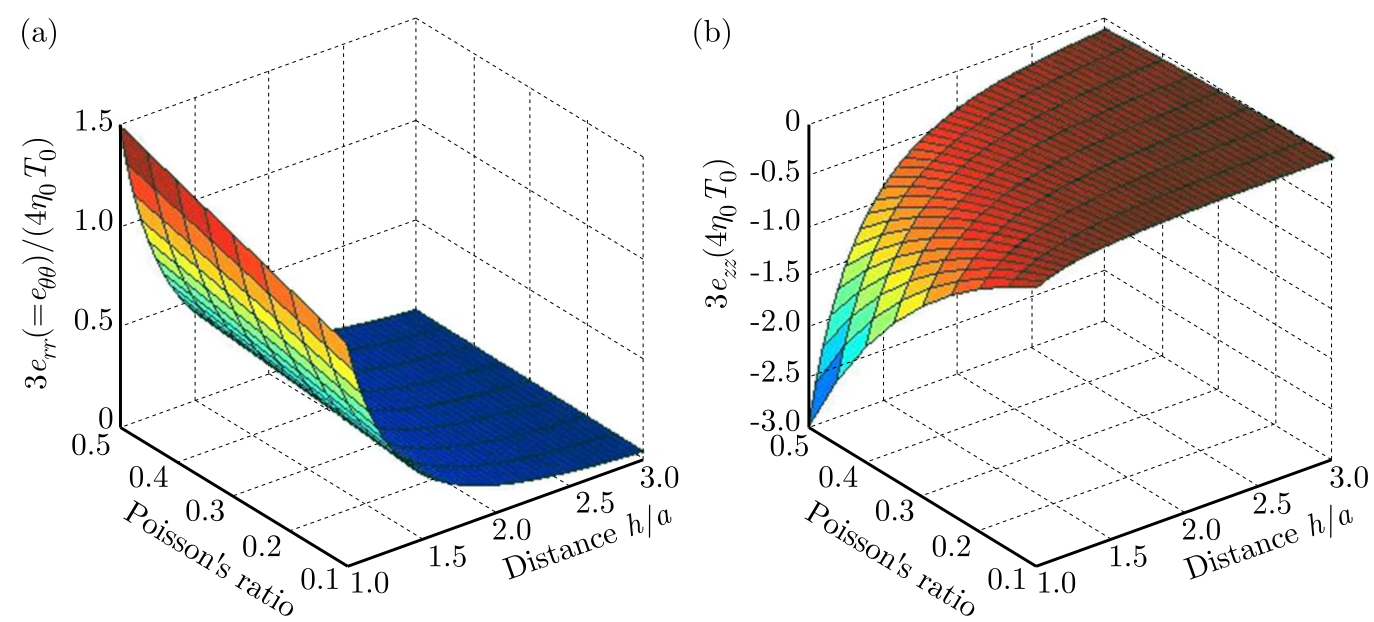

Fig. 2. (a) Strain component $e_{r r}\left(=e_{\theta \theta}\right)$ at the point $(0,0)$ for exterior points of the spherical inclusion, (b) strain component $e_{z z}$ atthe point $(0,0)$ for exterior points of the spherical inclusion
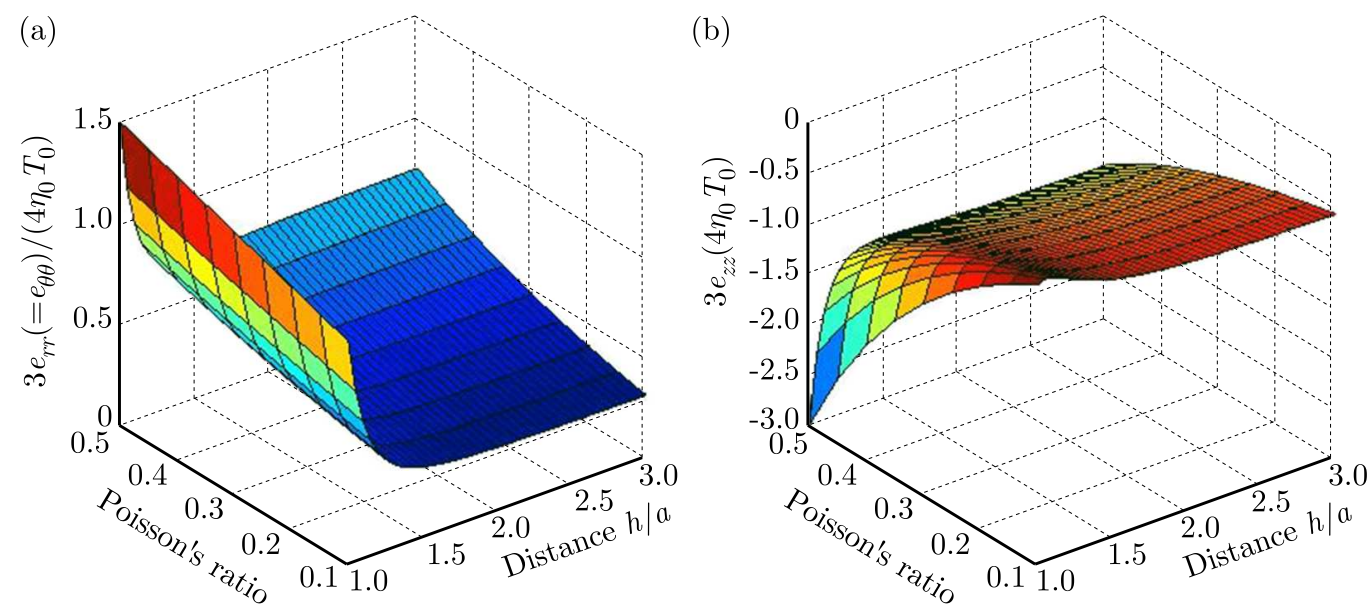

Fig. 3. (a) Strain component $e_{r r}\left(=e_{\theta \theta}\right)$ at the point $(0, h-a)$ just outside the spherical inclusion for exterior points, (b) strain component $e_{z z}$ at the point $(0, h-a)$ just outside the spherical inclusion for exterior points

Figure 4 shows the variation of the stress component $\sigma_{r r}\left(=\sigma_{\theta \theta}\right)$ at the point $(0,0)$ for exterior points of the spherical inclusion in the thermoelastic half-space. From Fig. 4, we observe that the stress component $\sigma_{r r}\left(=\sigma_{\theta \theta}\right)$ decreases gradually as the distance $h / a$ increases and it vanishes at infinitely large of $h / a$. Also, as Poisson's ratio $\nu$ increases from 0.1 to 0.5 , this stress increases 
quantitatively as shown in the figure. Figures $5 \mathrm{a}$ and $5 \mathrm{~b}$, respectively, show the variation of the stress component $\sigma_{r r}\left(=\sigma_{\theta \theta}\right)$ and $\sigma_{z z}$ at the point $(0, h-a)$ just outside the spherical inclusion in the thermoelastic half-space. From Fig. 5a, we observe that the stress component $\sigma_{r r}\left(=\sigma_{\theta \theta}\right)$ also decreases gradually with the increasing distance $h / a$ but it assumes finite positive values (depending on Poisson's ratio) at infinitely large values of the distance $h / a$. From Fig. 5b, we observe that as Poisson's ratio increases from 0.1 to 0.5 , the stress decreases more rapidly with increasing values of the distance $h / a$ and tends to finite negative values (depending on Poisson's ratio) at infinitely large values of the distance $h / a$.

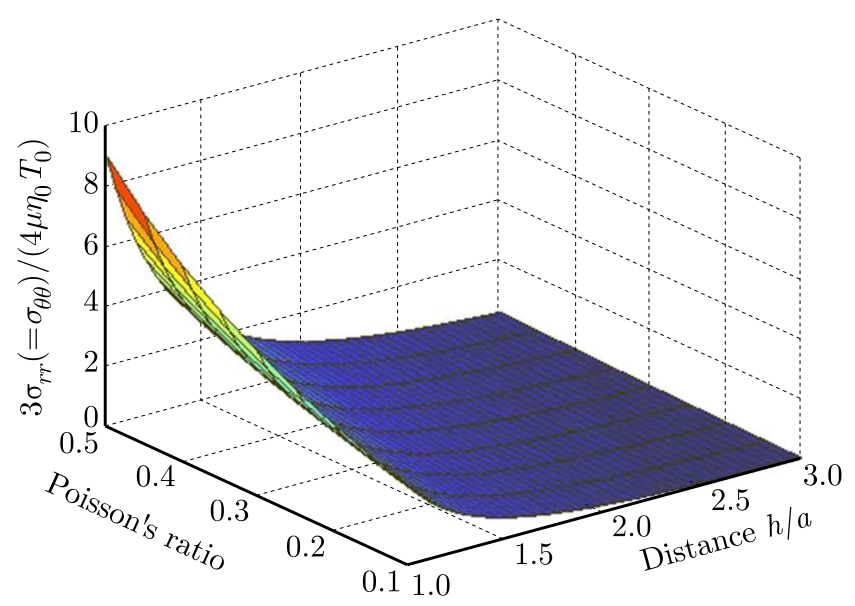

Fig. 4. Stress component $\sigma_{r r}\left(=\sigma_{\theta \theta}\right)$ at the point $(0,0)$ for exterior points of the spherical inclusion

(a)

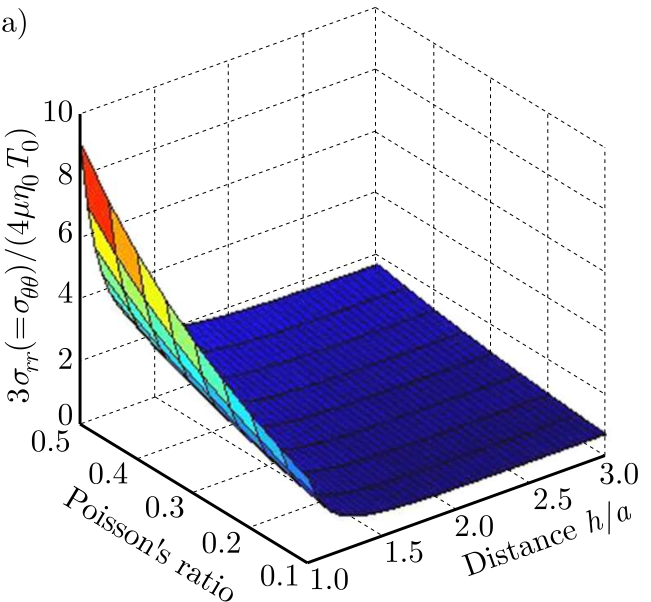

(b)

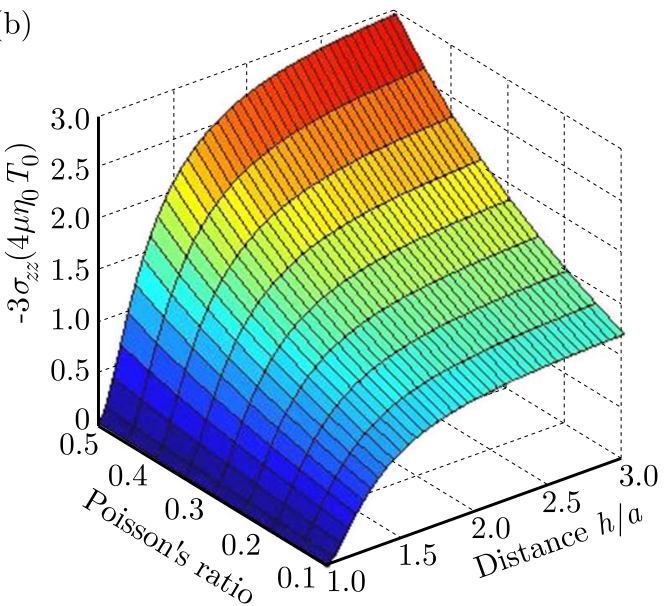

Fig. 5. (a) Stress component $\sigma_{r r}\left(=\sigma_{\theta \theta}\right)$ at the point $(0, h-a)$ just outside the spherical inclusion for exterior points, (b) stress component $\sigma_{z z}$ at the point $(0, h-a)$ just outside a spherical inclusion for exterior points

Figures $6 \mathrm{a}$ and $6 \mathrm{~b}$, respectively, show the variation of strain components $e_{r r}\left(=e_{\theta \theta}\right)$ and $e_{z z}$ at the point $(0, h)$ for interior points of the spherical inclusion. As observed from Fig. 6a, the strain $e_{r r}\left(=e_{\theta \theta}\right)$ increases continuously with the increasing distance $h / a$ and with increasing values of Poisson's ratio $\nu$ from 0.1 to 0.5 . From Fig. 6b, we observe that the strain $e_{z z}$ decreases gradually for all values of Poisson's ratio $\nu=0.1$ to 0.5 as the distance $h / a$ increases. It is noticed from Figs. 6a and 6b that these strains assume finite positive values (depending on Poisson's ratio) at infinitely large values of the distance $h / a$. Figures $7 \mathrm{a}$ and $7 \mathrm{~b}$, respectively, show the variation of stress components $\sigma_{r r}\left(=\sigma_{\theta \theta}\right)$ and $\sigma_{z z}$ at the point $(0, h)$ for interior points of the spherical inclusion. It is noticed from Figs. $7 \mathrm{a}$ and $7 \mathrm{~b}$ that the stresses decrease continuously 
as the distance $h / a$ increases and these assume finite negative values (depending on Poisson's ratio) at infinitely large values of distance $h / a$.
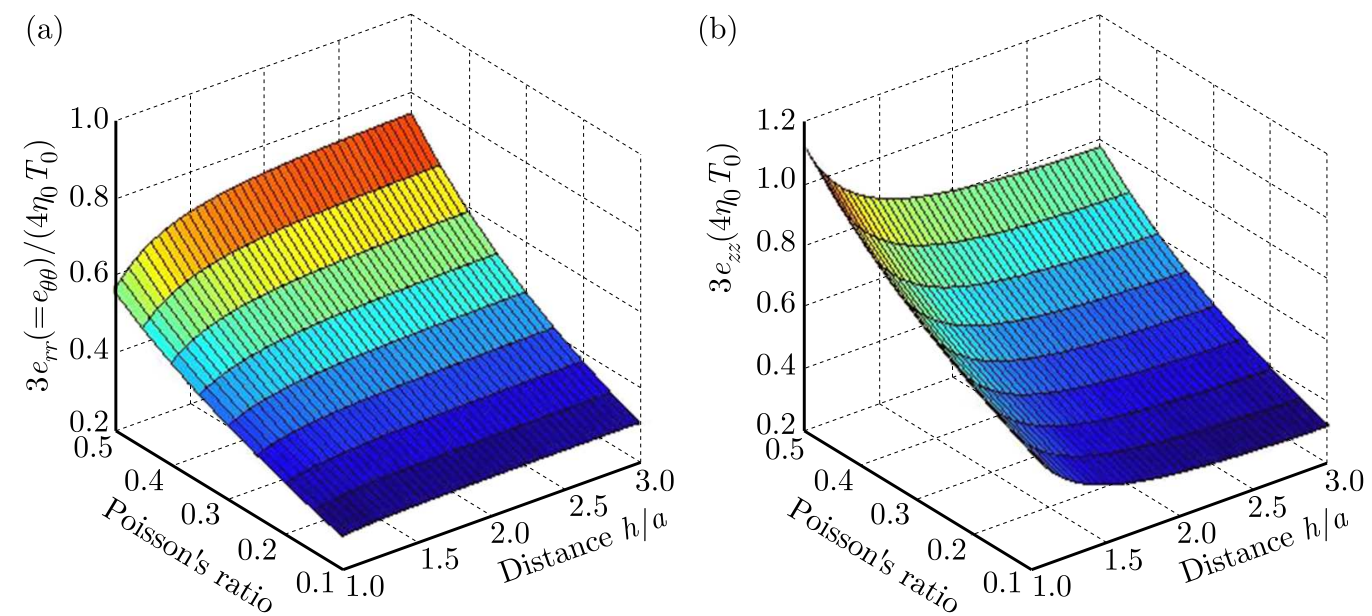

Fig. 6. (a) Strain component $e_{r r}\left(=e_{\theta \theta}\right)$ at the point $(0, h)$ for interior points of the spherical inclusion,

(b) strain component $e_{z z}$ at the point $(0, h)$ for interior points of the spherical inclusion
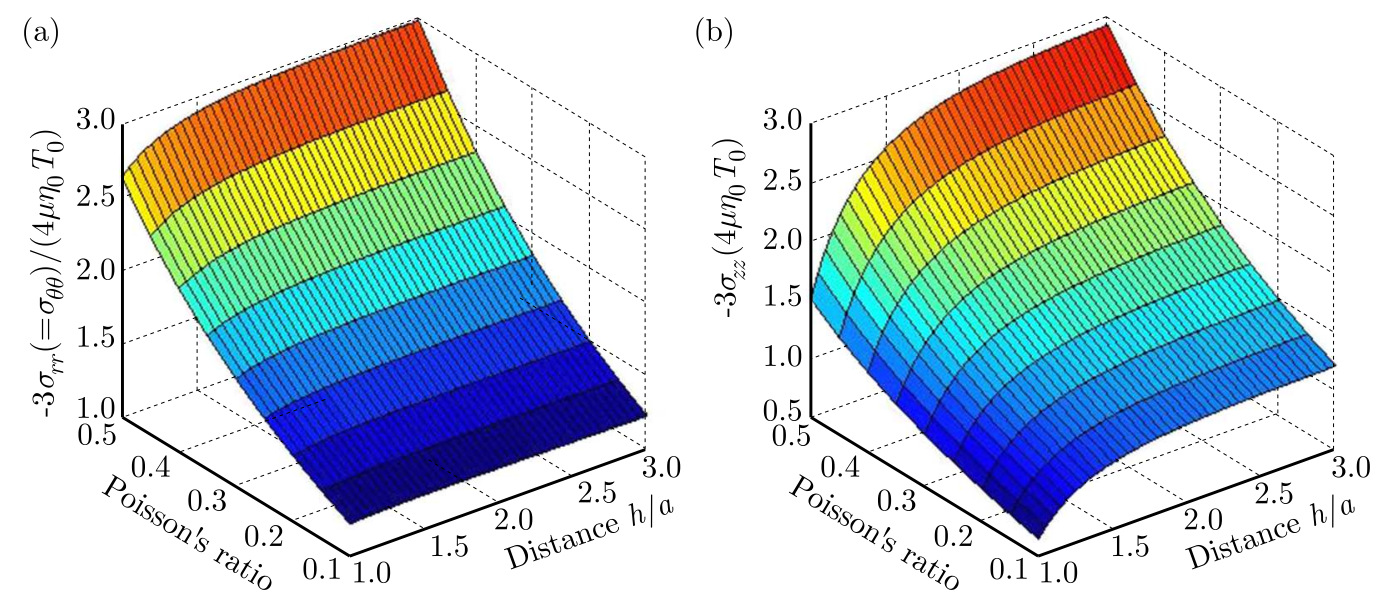

Fig. 7. (a) Stress component $\sigma_{r r}\left(=\sigma_{\theta \theta}\right)$ at the point $(0, h)$ for interior points of the spherical inclusion,

(b) stress component $\sigma_{z z}$ at the point $(0, h)$ for interior points of the spherical inclusion

\section{Conclusion}

In this paper, closed form analytical expressions for thermoelastic strain and stress components due to a spherical inclusion in a thermoelastic half-space are obtained. These expressions are derived in axisymmetric cylindrical coordinates in the context of steady-state uncoupled thermoelasticity using thermoelastic displacement potential functions. The thermoelastic strain and stress components for the exterior points of the spherical inclusion are the same as those of the center of dilatation. The variations of the thermoelastic strain and stress components for the exterior and interior points of the spherical inclusion are also shown graphically for different values of Poisson's ratios. It is observed that the strain and stress components at the points $(0, h-a)$ and $(0, h)$ approach finite values depending on Poisson's ratio at infinitely large values of the distance $h / a$, whereas at the point $(0,0)$ these quantities tend to zero as $h / a$ tends to infinity. Thus, Poisson's ratio has a significant effect on the strain and stress components due to the spherical inclusion in the thermoelastic half-space. 
Acknowledgement

We are thankful to the University Grants Commission, New Delhi, for financial support. We also thank the reviewer for useful suggestions in the improvement of our paper.

\section{References}

1. Barber J.R., 2002, Elasticity, Second Edition, Kluwer Academic Publishers, Dordrecht

2. Boley B.A., Weiner J.H., 1997, Theory of Thermal Stresses, Dover Publications, New York

3. Boresi A.P., Chong K.P., Lee J.D., 2011, Elasticity in Engineering Mechanics, Third Edition, John Wiley \& Sons, Inc., Hoboken, New Jersey

4. Davies J.H., 2003, Elastic field in a semi-infinite solid due to thermal expansion or a coherently misfitting inclusion, Journal of Applied Mechanics, 70, 5, 655-660

5. Duhamel J.M.C., 1837, Second mémoire sur les phénomènes thermo-mécaniques, Journal de l'Ecole Polytechnique, 15, 25, 1-57

6. Goodier J.N., 1937, On the integration of the thermoelastic equations, Philosophical Magazine, 7, 23, 1017-1032

7. Guell D.L., Dundurs J., 1967, Further results on center of dilatation and residual stresses in joined elastic half-spaces, [In:] Developments in Theoretical and Applied Mechanics, W.A. Shaw (Edit.), 3, 105-115, Pergamon Press, Oxford

8. Hu S.M., 1989, Stress from a parallelepipedic thermal inclusion in a semispace, Journal of Applied Physics, 66, 6, 2741-2743

9. Itou S., 2014, Plane strain and plane stress problems in thermoelasticity, [In:] Encyclopedia of Thermal Stresses, Richard B. Hetnarski (Eds.), Springer Netherlands, 3921-3930

10. Kolesov V.S., Vlasov N.M., Tisovskin L.O., Shatskin I.P., 1992, The stress-deformation state of an elastic half-space with a spheroidal thermal inclusion, International Applied Mechanics, 28, 7, 426-434

11. Kumagai H., Maeda Y., Ichihara M., Kame N., Kusakabe T., 2014, Seismic moment and volume change of a spherical source, Earth Planets and Space, 66, 7, 1-10

12. Leroux J., Fulleringer B., Nelias D., 2010, Contact analysis in presence of spherical inhomogeneities within a half-space, International Journal of Solids and Structures, 47, 3034-3049

13. Linzhi W., 2003, The elastic field induced by a hemispherical inclusion in the half-space, Acta Mechanica Sinica, 19, 3, 253-262

14. LiU S., WANG Q., 2005, Elastic fields due to eigenstrains in a half-space, Journal of Applied Mechanics, $\mathbf{7 2}, 871-878$

15. Mindlin R.D., Cheng D.H., 1950, Thermoelastic stress in the semi-infinite solid, Journal of Applied Physics, 21, 931-933

16. Neumann F., 1885, Vorlesungen über die Theorie der Elasticität der festen Körper und des Lichtäthers, B.G. Teubner, Leipzig

17. Nowacki W., 1957, State of stress in an infinite and semi-infinite elastic space due to an instantaneous source of heat, Bulletin de L'Academic Polonaise des Sciences, IV, 5, 2, 77-86

18. Nowacki W., 1986, Thermoelasticity, Second Edition, Pergamon Press, PWN-Polish Scientific Publishers, Warszawa

19. Nowinski J., 1961, Biharmonic solutions to the steady-state thermoelastic problems in three dimensions, Zeitschrift für angewandte Mathematik und Physik (ZAMP), XII, 2, 132-149

20. Nowinski J.L., 1963, A mean value theorem for an arbitrary steady-state thermoelastic problem for a solid sphere, Journal of the Society for Industrial and Applied Mathematics, 11, 3, 623-631 
21. Nowinski J.L., 1978, Theory of Thermoelasticity with Applications, Alphen Aan Den Rijn Sijthoff and Noordhoff International Publishers

22. SAdD M.H., 2005, Elasticity - Theory, Applications and Numerics, Elsevier Academic Press Inc., UK

23. SEN B., 1957, Note on a direct method of solving problems of elastic plates with circular boundaries having prescribed displacement, Kurze Mitteilungen-Brief Reports-Communications breves, VIII, 307-309

24. Sharma B.D., 1957, Stresses due to a nucleus of thermo-elastic strain (i) in an infinite elastic solid with spherical cavity and (ii) in a solid elastic sphere, Zeitschrift für angewandte Mathematik und Physik (ZAMP), VIII, 142-150

25. Timoshenko S., Goodier J.N., 1951, Theory of Elasticity, Second Edition, McGraw-Hill, New York

26. Truesdell C. (Edit.), 1984, Mechanics of Solids, Volume II, Linear Theories of Elasticity and Thermoelasticity, Springer-Verlag Berlin Heidelberg.

27. WAng M., HuAng K., 1991, Thermoelastic problems in the half space - An application of the general solution in elasticity, Applied Mathematics and Mechanics, 12, 9, 849-862

28. Yu H.Y., SAnday S.C., 1992, Centre of dilatation and thermal stresses in an elastic plate, Proceedings of the Royal Society of London A, 438, 103-112

29. Yu H.Y., Sanday S.C., Rath B.B., 1992, Thermoelastic stresses in bimaterials, Philosophical Magazine A, 65, 5, 1049-1064

30. Yu J.H., Kuang Z.B., Gu M.Y., 2002, Thermal stresses in joined semi-infinite solids, Journal of Thermal Stresses, 25, 9, 889-905

31. Zhou K., Chen W.W., Keer L.M., Wang Q. J., 2009, A fast method for solving threedimensional arbitrarily shaped inclusions in a half space, Computer Methods in Applied Mechanics and Engineering, 198, 9-12, 885-892 